\title{
Diversidade de moluscos em riachos de uma região de encosta no extremo sul do Brasil
}

\author{
Roger Lopes de Sá ${ }^{1}$, Luciani Santiñ ${ }^{2}$ Aline Monique Blank do Amaral', \\ Alcemar Rodrigues Martello ${ }^{1}$ \& Carla Bender Kotzian ${ }^{3,4}$ \\ ${ }^{1}$ Curso de Pós-graduação em Biodiversidade Animal, Universidade Federal de Santa Maria - UFSM, \\ CEP 97105-900, Santa Maria, RS, Brasil \\ ${ }^{2}$ Curso de Ciências Biológicas, Universidade Federal de Santa Maria-UFSM, \\ CEP 97105-900, Santa Maria, RS, Brasil \\ ${ }^{3}$ Programa de Pós-graduação em Biodiversidade Animal, Universidade Federal de Santa Maria - UFSM, \\ CEP 97105-900, Santa Maria, RS, Brasil \\ ${ }^{4}$ Autor para correspondência: Carla Bender Kotzian, e-mail: modrizralok@hotmail.com
}

SÁ, R.L., SANTIN, L., AMARAL, A.M.B., MARTELLO, A.R. \& KOTZIAN, C.B. Diversity of mollusks in streams of a montane region in southern Brazil. Biota Neotrop. 13(3): http://www.biotaneotropica.org.br/ v13n3/en/abstract?inventory+bn00213032013

\begin{abstract}
Stream mollusks in a region of slope in southern Brazil (Toropi River Basin) were inventoried by means of collections conducted in 40 sites, in altitudes ranging from 70 to 500 meters. At a whole, 18 species were found, represented predominantly by gastropods (11 species, $89.5 \%$ of the individuals). Potamolithus catharinae and Uncancylus concentricus were the dominant species. The latter was also well distributed, occurring in $81 \%$ of the sampling sites. Among the bivalves, only the invasive Asian clam Corbicula fluminea was abundant, but it occurred in only one sampling site. Four species were rare, being represented by less than three individuals, and the Unionoida were represented by two species only. Dominance and diversity of gastropods were higher than the bivalves, reflecting the gravel substrate of stream beds in the Toropi River Basin. The low richness recorded in the area studied is related to paucity of fine sediments and macrophytes in the streams, factors that commonly favors mollusks diversity. This condition also characterizes other southern Brazilian streams.

Keywords: freshwater mollusks, rithral zone, South America, gastropods, bivalves.
\end{abstract}

SÁ, R.L., SANTIN, L., AMARAL, A.M.B., MARTELLO, A.R. \& KOTZIAN, C.B. Diversidade de moluscos em riachos de uma região de encosta no extremo sul do Brasil. Biota Neotrop. 13(3): http://www.biotaneotropica. org.br/v13n3/pt/abstract?inventory+bn00213032013

Resumo: Moluscos de riachos de uma região de encosta, no extremo sul do Brasil (Bacia do Rio Toropi) foram inventariados através de coletas conduzidas em 40 locais, com altitudes variando entre 70 e 500 metros. Ao todo, 18 espécies foram encontradas, representadas predominantemente por gastrópodes (11 espécies, 89,5\% dos indivíduos). Potamolithus catharinae e Uncancylus concentricus foram as espécies dominantes. Aúltima espécie também foi a melhor distribuída, estando presente em $81 \%$ dos locais amostrados. Entre os bivalves, apenas a espécie asiática invasora Corbicula fluminea foi abundante, mas ocorreu em um único local de coleta. Cerca de quatro espécies foram raras, estando representadas por não mais que três indivíduos. Apenas duas espécies de Unionoida foram encontradas. A dominância e a maior diversidade de gastrópodes em relação ao registrado para bivalves reflete o substrato predominantemente cascalhoso dos riachos da Bacia do Rio Toropi. A baixa riqueza registrada na área de estudo pode ser atribuída à escassez de sedimentos finos e de macrófitas, fatores que costumam favorecer a diversidade de moluscos. Essa condição também caracteriza outros riachos do sul do Brasil. Palavras-chave: moluscos límnicos, zona ritral, América do Sul, gastrópodes, bivalves. 


\section{Introdução}

Moluscos límnicos encontram-se entre os grupos animais mais ameaçados de extinção (Ricciardi \& Rasmussen 1999, Bogan 2008, Strong et al. 2008). Práticas agrícolas como canalizações, barramentos, drenagem e liberação de agrotóxicos na água estão entre as principais causas do declínio da malacofauna de rios, lagos e banhados (Allan \& Flecker 1993, Allan 2004), além de certos poluentes (Dudgeon et al. 2006). Essas interferências antrópicas alteram fatores ambientais importantes para sobrevivência dos moluscos, como velocidade da água, conteúdo de oxigênio dissolvido, matéria orgânica em suspensão, etc. (e.g., Howard \& Cuffey 2006, Allen \& Vaughn 2010, Pérez-Quintero 2012). A introdução de espécies exóticas de moluscos também tem sido sugerida como causa importante para a extirpação de certas espécies nativas (Ricciardi et al. 1998, Strayer 1999, Mansur et al. 2012). Desta forma, estudar a biodiversidade dos moluscos límnicos, gerando informações sobre diversos aspectos da ecologia de espécies, populações e comunidades, é imprescindível para preservá-los (Lysne et al. 2008, Downing et al. 2010, PérezQuintero 2011).

A malacofauna dulcícola do estado do Rio Grande Sul, possivelmente, é uma das mais bem conhecidas no Brasil. Cerca de 373 espécies nativas (Simone 2006) e nove exóticas (Santos et al. 2012) são registradas no país. Para o Rio Grande do Sul, inventários indicam a presença de aproximadamente 95 espécies nativas e cinco exóticas (Simone 2006, Agudo-Padrón 2009). No Livro Vermelho da Fauna Brasileira Ameaçada de Extinção do estado 11 espécies são consideradas em perigo ou vulneráveis (Mansur et al. 2003). Adicionalmente, um dos principais obstáculos ao conhecimento da diversidade da malacofauna do estado é a falta de conhecimento sobre a distribuição das espécies (Mansur et al. 2003). Enquanto a fauna de banhados e lagoas é relativamente bem inventariada (Mansur et al. 1991, Veitenheimer-Mendes et al. 1992, Lanzer 2001, Drügg-Hahn et al. 2006), a maioria dos estudos conduzidos em rios restringe-se a certas bacias ou sub-bacias hidrográficas (Pereira et al. 2000a, b, Mansur \& Pereira 2006, Martello et al. 2006), ou a certos cursos de grandes rios (Pfeifer \& Pitoni 2003, Mansur \& Pereira 2006, Castillo et al. 2007). Riachos, que costumam abrigar uma alta diversidade de moluscos em certas regiões do mundo (Strong et al. 2008), são pouco estudados, principalmente em áreas de encosta (Pereira et al. 2011), as quais podem conter espécies endêmicas (Mehlhop \& Vaughn 1994). Além disso, a maioria não foi conduzida de forma exaustiva, ou seja, utilizando no mínimo 25 locais de amostragem, esforço recomendado para um bom conhecimento sobre as comunidades de moluscos (Dorazio 1999).

O uso dos recursos hídricos para produção de energia elétrica e principalmente para finalidades agrícolas no estado do Rio Grande do Sul é antigo (Beskow 1984). Reservatórios de diversas dimensões são extremamente numerosos e continuam proliferando. Açudes com tamanho igual ou inferior a 5 ha não necessitam de autorização de órgãos ambientais para serem construídos (Portaria SEMA/ FEPAM, n. 94, de 16 de dezembro de 2008), apesar de muitos deles serem obtidos às expensas dos barramentos de riachos. A situação de intenso represamento das águas de riachos, somada ao pouco conhecimento sobre a diversidade de moluscos nesses ambientes, mostra, claramente, a necessidade e a urgência de se inventariar esta malacofauna dos riachos do estado.

Este estudo apresenta o resultado de um levantamento de espécies de moluscos conduzido em 40 riachos da Bacia do Rio Toropi, na Encosta do Planalto do Rio Grande do Sul. Algumas características da estrutura da comunidade de moluscos também foram analisadas, de modo a contribuir-se de forma mais efetiva para o conhecimento da ecologia desse grupo, fornecendo informações que possam vir a ser úteis para ações de conservação.

\section{Material e Métodos}

\section{1. Área de estudo}

A Bacia do Rio Toropi localiza-se no extremo sul do Brasil (Estado do Rio Grande do Sul), entre as latitudes de $28^{\circ} 30^{\prime} \mathrm{S}$ a $31^{\circ} \mathrm{S}$, e longitudes de $53^{\circ} 30$ a $57^{\circ} \mathrm{W}$ (Figura 1), abrangendo uma área total de $47.740 \mathrm{~km}^{2}$. As nascentes localizam-se no Planalto Meridional, onde a altitude alcança 499 metros, mas a maioria dos rios e riachos ocupa a encosta do Planalto. O Rio Toropi é de $6^{\mathrm{a}}$ ordem próximo à foz, e desemboca no Rio Ibicuí, na Depressão Central, uma região com altitudes baixas, em torno de 72,54 metros (Hundertmarck \& Miorin 2001). Geologicamente, nas áreas mais altas, os rios correm sobre rochas vulcânicas da Formação Serra Geral, e na encosta correm sobre uma mistura de rochas vulcânicas e sedimentares, as últimas representadas pelas formações Botucatu e Caturrita. Próximo à foz, predominam sedimentos aluviais (Robaina et al. 2010).

O clima na região se caracteriza por apresentar chuvas durante todos os meses do ano, e precipitação acumulada anual variando entre 1.250 e $2.000 \mathrm{~mm}$ (Silva et al. 2006, Buriol et al. 2007). A temperatura média do mês mais quente é superior a $22^{\circ} \mathrm{C}$ e a do mês mais frio superior a $3{ }^{\circ} \mathrm{C}$ e, na região, o clima é considerado temperado (Maluf 2000). A vegetação da área estudada está inserida na zona de transição entre a Floresta Estacional Decidual na encosta do Planalto e a Savana (campos) na Depressão Central (Marcuzzo et al. 1998, Quadros \& Pillar 2002). Atualmente, grande parte da vegetação existente na Depressão Central deu lugar à agricultura (arroz), principalmente ao longo das margens dos rios e riachos (Pedron et al. 2006). $\mathrm{Na}$ encosta, plantações de milho e feijão são comuns, mas o cultivo de soja e a pecuária predominam, principalmente próximo ao Planalto.

\section{Locais de coleta}

As coletas foram feitas nos meses de outubro e novembro de 2010. Quarenta locais foram escolhidos para estudo (Figura 1), em riachos de ordem variável $\left(1^{\mathrm{a}}\right.$ a $\left.4^{\mathrm{a}}\right)$ e mata ciliar preservada, desde o Planalto até as áreas mais baixas da Depressão Central. A caracterização dos mesmos foi feita considerando-se a presença de

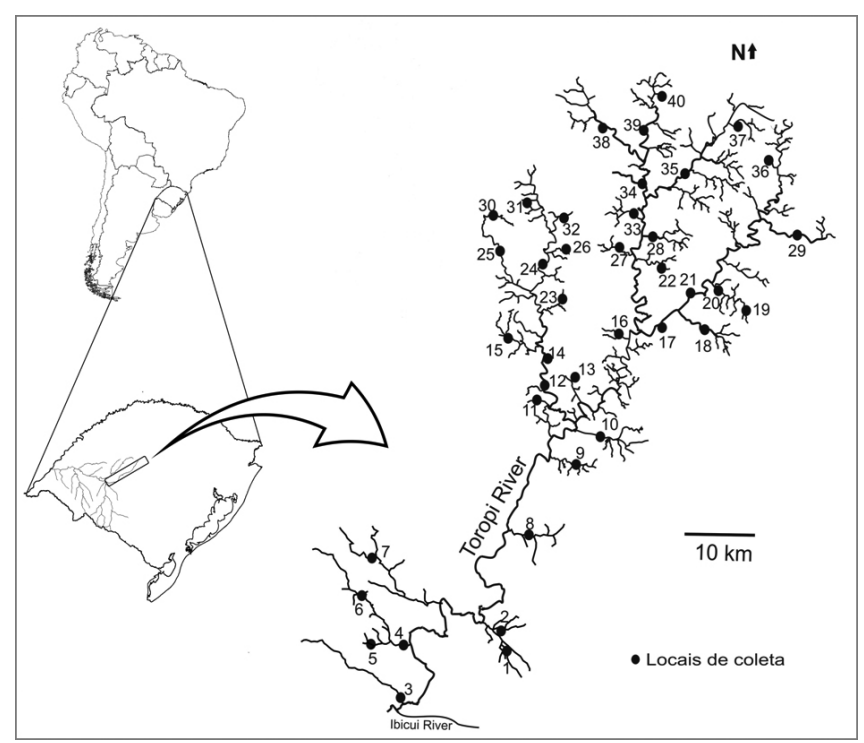

Figura 1. Área de estudo e locais de coleta de moluscos na bacia do Rio Toropi, RS, Brasil.

Figure 1. Study area and location of the mollusk sampling sites in the Toropi River Basin, RS, Brazil. 
macrófitas (identificadas até gênero), granulometria predominante ( $\geq 70 \%$, escala de Wentworth), altitude (determinada com auxílio de GPS) e ordem (determinada com base em mapas cartográficos com escala 1:50.000). A descrição ambiental de cada local encontra-se na Tabela 1.

\section{Amostragem e identificação}

As coletas foram realizadas, preferencialmente, nas margens dos rios, não se ultrapassando 1 metro de profundidade. As amostragens foram feitas através de parcelas de $5 \times 1 \mathrm{~m}\left(5 \mathrm{~m}^{2}\right)$, utilizando-se peneiras (malha $=1 \mathrm{~mm})($ Cummins 1994, Martello et al. 2006, Tietze \& De Francesco 2010). Em cada parcela, a amostragem foi conduzida por três pessoas igualmente treinadas, com esforço de coleta de 20 minutos/pessoa. Os exemplares coletados foram acondicionados em frascos com álcool 80\%. Macrófitas presentes no interior da área de cada parcela foram vistoriadas individualmente, e os moluscos nelas encontrados adicionados às amostras. No laboratório, a identificação foi feita até o nível de espécie, com auxílio de bibliografia especializada e, eventualmente, de especialistas. A classificação das espécies em categorias taxonômicas superiores

Tabela 1. Localização e caracterização dos locais de coleta de moluscos em riachos da bacia do Rio Toropi, RS, Brasil. (Granulometria: Ca = cascalho; $\mathrm{Ar}=$ areia; La = lama. Macrófitas: $1=$ gramínea; $2=$ junco; $3=$ Eleocharis $\mathrm{sp}$; $4=$ Litter $\mathrm{sp} . ; 5=$ Ludwigia $\mathrm{sp}$.; $6=$ Podostemun $\mathrm{sp}$.; $7=$ Eichhornia azurea; $8=$ Myriophyllum aquaticum $; 9=$ Pontederia lanceolata $; 10=$ Sagittaria montevidensis).

Table 1. Location and characterization of the mollusk sampling sites in streams of the Toropi River Basin, RS, Brazil. $(\mathrm{Granulometry:} \mathrm{Ca}=$ gravel; Ar $=$ sand; La = mud. Macrophytes: 1 = grasses; 2 = bulrush; $3=$ Eleocharis sp.; 4 =Litter sp.; $5=$ Ludwigia sp.; $6=$ Podostemun $\mathrm{sp}$.; $7=$ Eichhornia azurea (Sw.) Kunth; $8=$ Myriophyllum aquaticum (Vell.) Verdc.; $9=$ Pontederia lanceolata Nutt.; $10=$ Sagittaria montevidensis Cham. \& Schl.).

\begin{tabular}{|c|c|c|c|c|c|}
\hline Local & Localização geográfica & Altitude (m) & Ordem & Granulometria (\%) & Macrófitas \\
\hline 1 & $29^{\circ} 36^{\prime} 8.58 \mathrm{~S} / 54^{\circ} 28^{\prime} 58.6 \mathrm{~W}$ & 100 & $1^{\mathrm{a}}$ & $\mathrm{Ca}(89,5)$ & 1,2 \\
\hline 2 & $29^{\circ} 36^{\prime} 50.0 \mathrm{~S} / 54^{\circ} 20^{\prime} 52.3 \mathrm{~W}$ & 90 & $1^{\mathrm{a}}$ & $\mathrm{Ca}(87,8)$ & 8,10 \\
\hline 3 & $29^{\circ} 33^{\prime} 31.4 \mathrm{~S} / 54^{\circ} 29^{\prime} 09.2 \mathrm{~W}$ & 99 & $2^{\mathrm{a}}$ & $\mathrm{Ca}(95,3)$ & 5 \\
\hline 4 & $29^{\circ} 36^{\prime} 42.3 \mathrm{~S} / 54^{\circ} 21^{\prime} 04.2 \mathrm{~W}$ & 81 & $2^{\mathrm{a}}$ & $\operatorname{Ar}(41,9) \operatorname{La}(58,1)$ & 3 \\
\hline 5 & $29^{\circ} 35^{\prime} 07.5 \mathrm{~S} / 54^{\circ} 29^{\prime} 20.2 \mathrm{~W}$ & 100 & $3^{\mathrm{a}}$ & $\operatorname{Ar}(99,4)$ & - \\
\hline 6 & $29^{\circ} 36^{\prime} 37.9 \mathrm{~S} / 54^{\circ} 20^{\prime} 40.3 \mathrm{~W}$ & 94 & $3^{\mathrm{a}}$ & $\operatorname{Ar}(92,9)$ & 9 \\
\hline 7 & $29^{\circ} 38^{\prime} 35.4 \mathrm{~S} / 54^{\circ} 29^{\prime} 06.4 \mathrm{~W}$ & 96 & $4^{\mathrm{a}}$ & $\operatorname{Ar}(91,8)$ & - \\
\hline 8 & $29^{\circ} 43^{\prime} 00.5 \mathrm{~S} / 54^{\circ} 30^{\prime} 28.5 \mathrm{~W}$ & 92 & $4^{\mathrm{a}}$ & $\mathrm{La}(100)$ & 5,7 \\
\hline 9 & $29^{\circ} 33^{\prime} 31.1 \mathrm{~S} / 54^{\circ} 29^{\prime} 09.8 \mathrm{~W}$ & 143 & $1^{\mathrm{a}}$ & $\mathrm{Ca}(59,2) \mathrm{Ar}(40,2)$ & - \\
\hline 10 & $29^{\circ} 31^{\prime} 30.9 \mathrm{~S} / 54^{\circ} 13^{\prime} 56.6 \mathrm{~W}$ & 116 & $1^{\mathrm{a}}$ & $\mathrm{Ca}(38,4) \operatorname{Ar}(60,6)$ & - \\
\hline 11 & $29^{\circ} 26^{\prime} 31.7 \mathrm{~S} / 54^{\circ} 12^{\prime} 48.8 \mathrm{~W}$ & 170 & $2^{\mathrm{a}}$ & $\mathrm{Ca}(73,6)$ & - \\
\hline 12 & $29^{\circ} 27^{\prime} 13.0 \mathrm{~S} / 54^{\circ} 09^{\prime} 55.1 \mathrm{~W}$ & 158 & $2^{\mathrm{a}}$ & $\mathrm{Ca}(78,1)$ & - \\
\hline 13 & $29^{\circ} 21^{\prime} 00.4 \mathrm{~S} / 54^{\circ} 06^{\prime} 09.0 \mathrm{~W}$ & 159 & $3^{\mathrm{a}}$ & $\mathrm{Ca}(58) \operatorname{Ar}(39,9)$ & 4,6 \\
\hline 14 & $29^{\circ} 28^{\prime} 39.7 \mathrm{~S} / 54^{\circ} 14^{\prime} 00.9 \mathrm{~W}$ & 106 & $3^{\mathrm{a}}$ & $\operatorname{Ar}(90,2)$ & - \\
\hline 15 & $29^{\circ} 27^{\prime} 40.2 \mathrm{~S} / 54^{\circ} 11^{\prime} 37.9 \mathrm{~W}$ & 134 & $4^{\mathrm{a}}$ & $\mathrm{Ca}(96,6)$ & 6 \\
\hline 16 & $29^{\circ} 30^{\prime} 56.5 \mathrm{~S} / 54^{\circ} 09^{\prime} 29.3 \mathrm{~W}$ & 110 & $4^{\mathrm{a}}$ & $\operatorname{Ar}(93,3)$ & - \\
\hline 17 & $29^{\circ} 31^{\prime} 18.5 \mathrm{~S} / 54^{\circ} 06^{\prime} 02.2 \mathrm{~W}$ & 230 & $1^{\mathrm{a}}$ & $\mathrm{Ca}(93,5)$ & - \\
\hline 18 & $29^{\circ} 32^{\prime} 34.0 \mathrm{~S} / 54^{\circ} 08^{\prime} 15.4 \mathrm{~W}$ & 227 & $1^{\mathrm{a}}$ & $\mathrm{Ca}(91,4)$ & - \\
\hline 19 & $29^{\circ} 25^{\prime} 06.8 \mathrm{~S} / 54^{\circ} 05^{\prime} 29.4 \mathrm{~W}$ & 231 & $2^{\mathrm{a}}$ & $\mathrm{Ca}(100)$ & - \\
\hline 20 & $29^{\circ} 32^{\prime} 53.8 \mathrm{~S} / 54^{\circ} 09^{\prime} 06.2 \mathrm{~W}$ & 221 & $2^{\mathrm{a}}$ & $\mathrm{Ca}(64,5) \operatorname{Ar}(34,4)$ & - \\
\hline 21 & $29^{\circ} 21^{\prime} 00.8 \mathrm{~S} / 54^{\circ} 12^{\prime} 37.7 \mathrm{~W}$ & 267 & $3^{\mathrm{a}}$ & $\mathrm{Ca}(100)$ & - \\
\hline 22 & $29^{\circ} 26^{\prime} 54.5 \mathrm{~S} / 54^{\circ} 07^{\prime} 46.3 \mathrm{~W}$ & 206 & $3^{\mathrm{a}}$ & $\mathrm{Ca}(95,7)$ & - \\
\hline 23 & $29^{\circ} 19^{\prime} 06.2 \mathrm{~S} / 54^{\circ} 09^{\prime} 15.7 \mathrm{~W}$ & 274 & $4^{\mathrm{a}}$ & $\mathrm{Ca}(49,7) \operatorname{Ar}(49,1)$ & 4,6 \\
\hline 24 & $29^{\circ} 28^{\prime} 59.3 \mathrm{~S} / 54^{\circ} 04^{\prime} 18.1 \mathrm{~W}$ & 214 & $4^{\mathrm{a}}$ & $\mathrm{Ca}(96,4)$ & - \\
\hline 25 & $29^{\circ} 16^{\prime} 57.2 \mathrm{~S} / 54^{\circ} 05^{\prime} 57.7 \mathrm{~W}$ & 377 & $1^{\mathrm{a}}$ & $\mathrm{Ca}(95,2)$ & - \\
\hline 26 & $29^{\circ} 19^{\prime} 35.9 \mathrm{~S} / 54^{\circ} 03^{\prime} 28.6 \mathrm{~W}$ & 385 & $1^{\mathrm{a}}$ & $\operatorname{Ar}(94,9)$ & 6 \\
\hline 27 & $29^{\circ} 19^{\prime} 04.9 \mathrm{~S} / 54^{\circ} 07^{\prime} 20.0 \mathrm{~W}$ & 352 & $2^{\mathrm{a}}$ & $\mathrm{Ca}(97)$ & - \\
\hline 28 & $29^{\circ} 22^{\prime} 56.5 \mathrm{~S} / 54^{\circ} 03^{\prime} 46.6 \mathrm{~W}$ & 318 & $2^{\mathrm{a}}$ & $\operatorname{Ar}(88,5)$ & 6 \\
\hline 29 & $29^{\circ} 14^{\prime} 34.9 \mathrm{~S} / 53^{\circ} 57^{\prime} 58.6 \mathrm{~W}$ & 353 & $3^{\mathrm{a}}$ & $\mathrm{Ca}(94)$ & - \\
\hline 30 & $29^{\circ} 11^{\prime} 27.2 \mathrm{~S} / 54^{\circ} 05^{\prime} 06.7 \mathrm{~W}$ & 391 & $3^{\mathrm{a}}$ & $\mathrm{Ca}(92,6)$ & - \\
\hline 31 & $29^{\circ} 06^{\prime} 19.4 \mathrm{~S} / 53^{\circ} 56^{\prime} 39.1 \mathrm{~W}$ & 396 & $4^{\mathrm{a}}$ & $\mathrm{Ca}(93,2)$ & - \\
\hline 32 & $29^{\circ} 26^{\prime} 42.0 \mathrm{~S} / 53^{\circ} 56^{\prime} 23.8 \mathrm{~W}$ & 327 & $4^{\mathrm{a}}$ & $\mathrm{Ca}(98,6)$ & - \\
\hline 33 & $29^{\circ} 18^{\prime} 37.2 \mathrm{~S} / 53^{\circ} 48^{\prime} 57.4 \mathrm{~W}$ & 435 & $1^{\mathrm{a}}$ & $\mathrm{Ca}(89,1)$ & - \\
\hline 34 & $29^{\circ} 09^{\prime} 30.8 \mathrm{~S} / 53^{\circ} 44^{\prime} 49.7 \mathrm{~W}$ & 476 & $1^{\mathrm{a}}$ & $\mathrm{Ca}(47) \operatorname{Ar}(51,2)$ & - \\
\hline 35 & $29^{\circ} 16^{\prime} 25.7 \mathrm{~S} / 53^{\circ} 47^{\prime} 56.8 \mathrm{~W}$ & 417 & $2^{\mathrm{a}}$ & $\mathrm{Ca}(91,6)$ & - \\
\hline 36 & $29^{\circ} 05^{\prime} 48.5 \mathrm{~S} / 53^{\circ} 58^{\prime} 29.3 \mathrm{~W}$ & 424 & $2^{\mathrm{a}}$ & $\mathrm{Ca}(90,8)$ & - \\
\hline 37 & $29^{\circ} 06^{\prime} 44.5 \mathrm{~S} / 53^{\circ} 50^{\prime} 06.2 \mathrm{~W}$ & 414 & $3^{\mathrm{a}}$ & $\mathrm{Ca}(92)$ & - \\
\hline 38 & $29^{\circ} 03^{\prime} 58.6 \mathrm{~S} / 53^{\circ} 56^{\prime} 26.1 \mathrm{~W}$ & 468 & $3^{\mathrm{a}}$ & $\mathrm{Ca}(89,7)$ & - \\
\hline 39 & $29^{\circ} 24^{\prime} 27.0 \mathrm{~S} / 53^{\circ} 42^{\prime} 57.3 \mathrm{~W}$ & 438 & $4^{\mathrm{a}}$ & $\mathrm{Ca}(95)$ & - \\
\hline 40 & $29^{\circ} 09^{\prime} 09.5 \mathrm{~S} / 53^{\circ} 58^{\prime} 05.8 \mathrm{~W}$ & 413 & $4^{\mathrm{a}}$ & $\mathrm{Ca}(98,2)$ & - \\
\hline
\end{tabular}


a gênero baseou-se em Simone (2006). O material-testemunha foi depositado na Coleção de Moluscos do Setor de Zoologia, Departamento de Biologia da Universidade Federal de Santa Maria (UFSM), sob a seguinte numeração: UFSM-M-101.371 Corbicula fluminea (Müller, 1774); UFSM-M-101.372 a 101.395 Uncancylus concentricus (d'Orbigny, 1835); UFSM-M-101.396 a 101.402 Potamolithus catharinae Pilsbry, 1911; UFSM-M-101.403 a 101.406 Hebetancylus moricandi (d'Orbigny, 1837); UFSM-M-101.407 a 101.415 Potamolithus ribeirensis Pilsbry, 1911; UFSM-M-101.416 a 101.419 Heleobia bertoniana Pilsbry, 1911; UFSM-M-101.420 a 101.423 Biomphalaria straminea (Dunker, 1848); UFSM-M-101.424 a 101.430 Biomphalaria tenagophila (d'Orbigny, 1835); UFSM-M-101.431 a 101.434 Lymnaea columella (Say, 1817); UFSM-M-101.435 e 101.436 Eupera klappenbachi Mansur \& Veitenheimer, 1975; UFSM-M-101.437 Eupera elliptica Ituarte \& Mansur, 1993; UFSM-M-101.438 a 101.441 Asolene spixii Orbigny, 1838; UFSM-M-101.442 a 101.444 Pisidium sterkianum Pilsbry, 1897; UFSM-M-101.445 Pisidium punctiferum (Guppy, 1867); UFSM-M-101.446 a 101.449 Pomacea canaliculata (Lamarck, 1804); UFSM-M-101.450 Pomella americanista (Ihering, 1919); UFSM-M-101.451 a 101.455 Diplodon rhuacoicus (d'Orbigny, 1835); e UFSM-M 101.460 Anodontites tenebricosus (Lea, 1834).

\section{Análise dos dados}

Exemplares representados apenas por conchas não foram incluídos nas análises. A diversidade da comunidade foi analisada conforme riqueza $(\mathrm{S})$, número $(\mathrm{N})$, frequência relativa $(\%)$ e dominância. A riqueza acumulada de espécies na área de estudo foi estimada através de curva do coletor, através do método de Coleman (Coleman 1981), obtida com 500 curvas geradas por adição aleatória das amostras, no programa EstimateS 8.0 (Cowell 2006). Este método foi selecionado, pois calcula a flutuação em torno da curva média, quando as amostras são adicionadas, e é excelente para avaliar o quanto o inventário se aproxima da riqueza total da área (Colwell \& Coddington 1994).

\section{Resultados}

$\mathrm{Na}$ área de estudo, 2300 exemplares vivos, classificados em 18 espécies foram encontrados. A curva do coletor, construída com base nos moluscos obtidos em cada local de coleta, não atingiu a assíntota, mas mostrou tendência à estabilização (Figura 2), sugerindo que algum acréscimo na riqueza poderá ser obtido com novos esforços de amostragem.

Os gastrópodes foram mais numerosos, representando 89,5\% do total de indivíduos coletados, e foram representados por 11 espécies (Tabela 2): Pomacea canaliculata, Asolene spixii, Pomella americanista, Potamolithus catharinae, Potamolithus ribeirensis, Heleobia bertoniana Lymnaea columella, Biomphalaria straminea, Biomphalaria tenagophila, Hebetancylus moricandi e Uncancylus concentricus, enquanto os bivalves foram representados por sete espécies: Diplodon rhuacoicus, Anodontites tenebricosus, Corbicula fluminea, Pisidium punctiferum, Pisidium sterkianum, Eupera elliptica e Eupera klappenbachi (Figuras 3 a 5).

\section{Discussão}

A riqueza $(S=18)$ e as espécies dominantes encontradas na área de estudo são semelhantes às registradas em outros riachos do sul do Brasil (Martello et al. 2006, Freitas 2011, Pereira et al. 2011). Riqueza maior ( $\mathrm{S}=23$ ) só foi registrada em uma área de nascentes do Rio Gravataí, localizada em banhado, rico em macrófitas (VeitenheimerMendes et al. 1992). A riqueza encontrada nos riachos do extremo sul do Brasil contrasta com aquela mencionada para outros locais

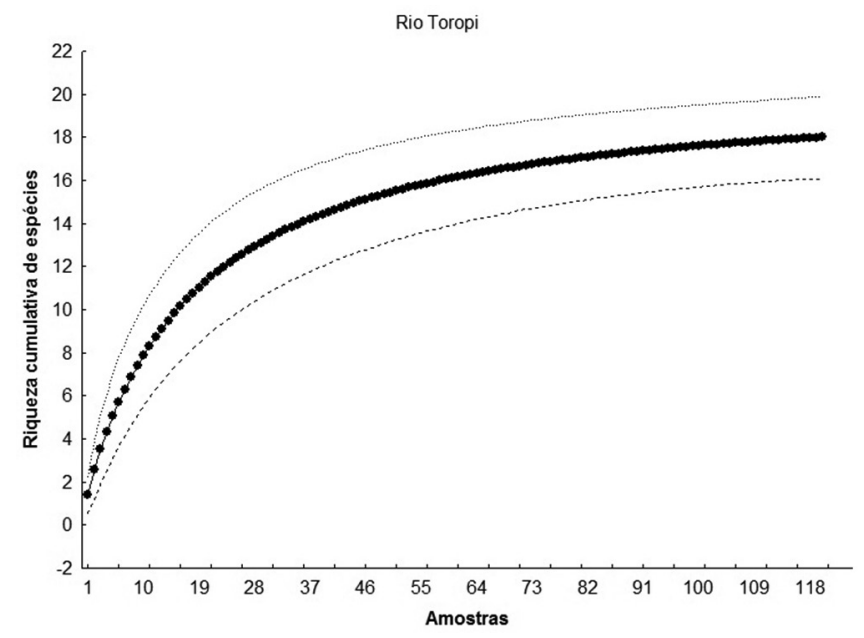

Figura 2. Curva do coletor com base nos moluscos obtidos em cada local de coleta da bacia do Rio Toropi, RS, Brasil.

Figure 2. Species accumulation curve based on mollusks collected in each sampling site in the Toropi River Basin, RS, Brazil.
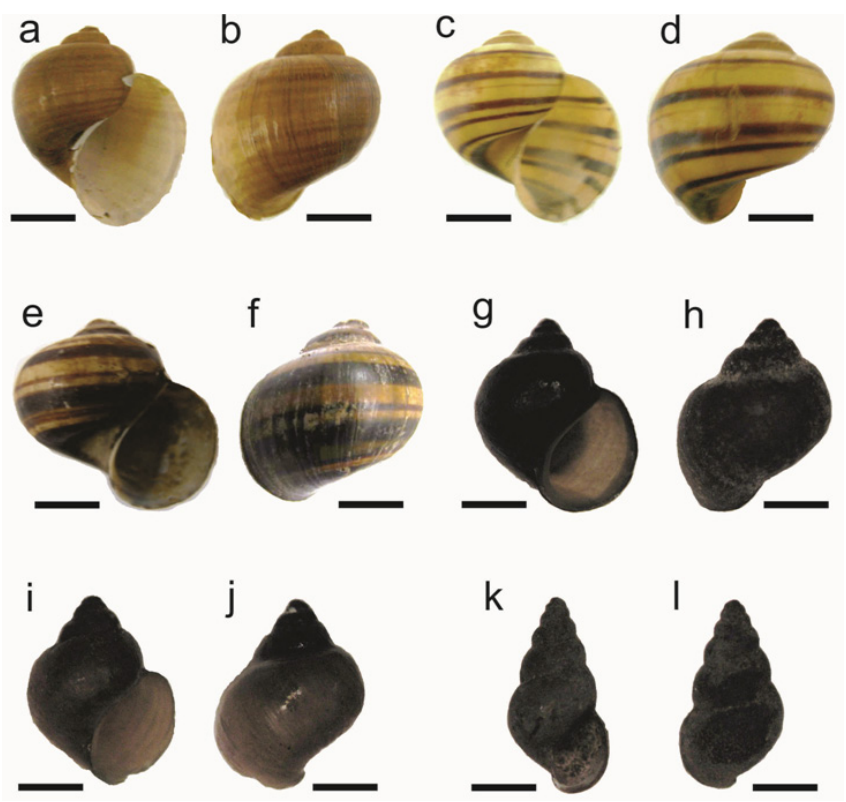

Figura 3. Pomacea canaliculata $(\mathrm{a}, \mathrm{b})$ vista ventral e dorsal $($ escala $=0,9 \mathrm{~cm})$; Asolene spixii $(\mathrm{c}, \mathrm{d})$ vista ventral e dorsal (escala $=0,9 \mathrm{~cm})$; Pomella americanista $(\mathrm{e}, \mathrm{f})$ vista ventral e dorsal (escala $=0,7 \mathrm{~cm}) ;$ Potamolithus catharinae $(\mathrm{g}, \mathrm{h})$ vista ventral e dorsal (escala $=0,2 \mathrm{~cm})$; Potamolithus ribeirensis $(\mathrm{i}, \mathrm{j})$ vista ventral e dorsal $(\mathrm{escala}=0,1 \mathrm{~cm})$; Heleobia bertoniana $(\mathrm{k}, 1)$ vista ventral e dorsal $($ escala $=0,4 \mathrm{~cm})$.

Figure 3. Pomacea canaliculata $(\mathrm{a}, \mathrm{b})$ ventral and dorsal view $(\mathrm{scale}=0.9 \mathrm{~cm})$; Asolene spixii $(\mathrm{c}, \mathrm{d})$ ventral and dorsal view $($ scale $=0.9 \mathrm{~cm}) ;$ Pomella americanista $(\mathrm{e}, \mathrm{f})$ ventral and dorsal view $(\mathrm{scale}=0.7 \mathrm{~cm}) ;$ Potamolithus catharinae $(\mathrm{g}, \mathrm{h})$ ventral and dorsal view (scale $=0.2 \mathrm{~cm}) ;$ Potamolithus ribeirensis $(\mathrm{i}, \mathrm{j})$ ventral and dorsal view $($ scale $=0.1 \mathrm{~cm})$; Heleobia bertoniana $(\mathrm{k}, 1)$ ventral and dorsal view $($ scale $=0.4 \mathrm{~cm})$.

do hemisfério Norte. Um estudo abrangente sobre a diversidade de gastrópodes mostra que riachos são locais com grande riqueza e endemismo (Strong et al. 2008). Com efeito, um número maior de espécies em relação ao aqui registrado só é observado em rios de maior porte e/ou em áreas mais baixas da região sul do Brasil, bem como em áreas vizinhas (Bonetto \& Di Persia 1975, Pereira et al. 
Tabela 2. Abundância (N) e riqueza (S) de espécies de moluscos encontradas em riachos da Bacia do Rio Toropi, RS, Brasil.

Table 2. Abundance (N) and richness (S) of mollusk species found in streams of the Toropi River Basin, RS, Brazil.

\begin{tabular}{|c|c|c|c|c|c|c|c|c|c|c|c|c|c|c|c|c|c|c|c|c|c|}
\hline \multirow{2}{*}{ Espécies } & \multicolumn{20}{|c|}{ Locais de coleta } & \multirow{2}{*}{ Total } \\
\hline & 1 & 2 & 3 & 4 & 5 & 6 & 7 & 8 & 9 & 10 & 11 & 12 & 13 & 14 & 15 & 16 & 17 & 18 & 19 & 20 & \\
\hline P. canaliculata & 13 & 11 & 0 & 0 & 2 & 0 & 1 & 3 & 0 & 0 & 0 & 0 & 0 & 0 & 0 & 0 & 0 & 0 & 0 & 0 & - \\
\hline A. spixii & 0 & 0 & 0 & 0 & 0 & 0 & 1 & 11 & 0 & 0 & 0 & 0 & 0 & 0 & 0 & 0 & 0 & 0 & 0 & 0 & - \\
\hline P. americanista & 0 & 0 & 0 & 0 & 0 & 0 & 0 & 0 & 0 & 0 & 0 & 0 & 0 & 0 & 1 & 0 & 0 & 0 & 0 & 0 & - \\
\hline P. catharinae & 0 & 0 & 0 & 0 & 0 & 1 & 0 & 0 & 0 & 0 & 0 & 4 & 0 & 0 & 0 & 0 & 0 & 0 & 17 & 0 & - \\
\hline P. ribeirensis & 0 & 0 & 0 & 0 & 0 & 0 & 0 & 0 & 0 & 0 & 0 & 12 & 0 & 2 & 0 & 0 & 0 & 0 & 5 & 0 & - \\
\hline H. bertoniana & 0 & 0 & 0 & 0 & 0 & 0 & 0 & 113 & 0 & 0 & 0 & 0 & 0 & 7 & 0 & 0 & 0 & 6 & 0 & 245 & - \\
\hline L. columella & 0 & 0 & 1 & 0 & 0 & 0 & 0 & 0 & 0 & 0 & 0 & 0 & 0 & 2 & 0 & 0 & 0 & 0 & 0 & 1 & - \\
\hline B. straminea & 0 & 0 & 0 & 0 & 0 & 0 & 0 & 20 & 0 & 0 & 0 & 0 & 0 & 0 & 0 & 0 & 0 & 0 & 0 & 0 & - \\
\hline B. tenagophila & 1 & 1 & 0 & 4 & 0 & 0 & 1 & 61 & 0 & 0 & 0 & 0 & 0 & 1 & 0 & 0 & 1 & 1 & 0 & 0 & - \\
\hline H. moricandi & 2 & 0 & 0 & 6 & 0 & 0 & 0 & 86 & 0 & 0 & 0 & 0 & 0 & 0 & 0 & 1 & 0 & 0 & 0 & 0 & - \\
\hline U. concentricus & 54 & 7 & 1 & 0 & 20 & 0 & 0 & 4 & 1 & 1 & 42 & 51 & 11 & 88 & 3 & 2 & 39 & 0 & 3 & 0 & - \\
\hline D. rhuacoicus & 0 & 0 & 0 & 0 & 0 & 0 & 6 & 0 & 0 & 0 & 0 & 0 & 0 & 0 & 1 & 1 & 0 & 0 & 0 & 0 & - \\
\hline A. tenebricosus & 0 & 0 & 0 & 0 & 0 & 1 & 0 & 0 & 0 & 0 & 0 & 0 & 0 & 0 & 1 & 0 & 0 & 0 & 0 & 0 & - \\
\hline C. fluminea & 0 & 0 & 0 & 0 & 0 & 0 & 149 & 0 & 0 & 0 & 0 & 0 & 0 & 0 & 0 & 0 & 0 & 0 & 0 & 0 & - \\
\hline P. punctiferum & 0 & 0 & 0 & 0 & 0 & 0 & 0 & 0 & 0 & 0 & 0 & 0 & 0 & 0 & 0 & 0 & 0 & 0 & 0 & 0 & - \\
\hline P. sterkianum & 16 & 22 & 0 & 0 & 0 & 0 & 0 & 11 & 0 & 0 & 0 & 0 & 0 & 1 & 0 & 0 & 0 & 2 & 0 & 0 & - \\
\hline E. elliptica & 0 & 0 & 0 & 0 & 0 & 0 & 0 & 0 & 0 & 0 & 0 & 0 & 0 & 0 & 0 & 0 & 0 & 0 & 0 & 0 & - \\
\hline E. klappenbachi & 0 & 0 & 0 & 0 & 0 & 0 & 0 & 17 & 0 & 0 & 0 & 0 & 0 & 0 & 0 & 0 & 0 & 0 & 0 & 0 & - \\
\hline $\mathbf{S}$ & 5 & 4 & 2 & 2 & 2 & 2 & 5 & 9 & 1 & 1 & 1 & 3 & 1 & 6 & 4 & 3 & 2 & 3 & 3 & 2 & - \\
\hline $\mathbf{N}$ & 86 & 41 & 2 & 10 & 22 & 2 & 158 & 326 & 1 & 1 & 42 & 67 & 11 & 101 & 6 & 4 & 40 & 9 & 25 & 246 & - \\
\hline \multirow{2}{*}{ Espécies } & \multicolumn{20}{|c|}{ Locais de coleta } & \multirow{2}{*}{ Total } \\
\hline & 21 & 22 & 23 & 24 & 25 & 26 & 27 & 28 & 29 & 30 & 31 & 32 & 33 & 34 & 35 & 36 & 37 & 38 & 39 & 40 & \\
\hline P. canaliculata & 0 & 0 & 0 & 0 & 0 & 0 & 0 & 0 & 0 & 0 & 0 & 0 & 0 & 0 & 0 & 0 & 0 & 0 & 0 & 0 & 30 \\
\hline A. spixii & 0 & 0 & 0 & 0 & 0 & 0 & 0 & 0 & 2 & 0 & 0 & 2 & 0 & 0 & 0 & 0 & 0 & 0 & 0 & 0 & 16 \\
\hline P. americanista & 0 & 0 & 0 & 0 & 0 & 0 & 0 & 0 & 0 & 0 & 0 & 0 & 0 & 0 & 0 & 0 & 0 & 0 & 0 & 0 & 1 \\
\hline P. catharinae & 0 & 0 & 22 & 0 & 0 & 0 & 23 & 0 & 153 & 0 & 0 & 161 & 0 & 0 & 0 & 0 & 0 & 0 & 8 & 95 & 484 \\
\hline P. ribeirensis & 0 & 0 & 28 & 0 & 0 & 0 & 0 & 1 & 107 & 0 & 0 & 95 & 0 & 0 & 0 & 0 & 0 & 0 & 2 & 80 & 332 \\
\hline H. bertoniana & 0 & 0 & 0 & 0 & 0 & 0 & 0 & 0 & 0 & 0 & 0 & 0 & 0 & 0 & 0 & 0 & 0 & 0 & 0 & 0 & 371 \\
\hline L. columella & 0 & 0 & 1 & 0 & 0 & 0 & 0 & 0 & 0 & 0 & 0 & 0 & 0 & 0 & 0 & 0 & 2 & 0 & 0 & 0 & 7 \\
\hline B. straminea & 0 & 0 & 0 & 0 & 0 & 0 & 0 & 0 & 1 & 0 & 0 & 3 & 0 & 0 & 0 & 0 & 0 & 0 & 0 & 0 & 24 \\
\hline B. tenagophila & 0 & 0 & 0 & 0 & 0 & 0 & 0 & 0 & 0 & 0 & 0 & 0 & 0 & 0 & 0 & 1 & 0 & 0 & 0 & 1 & 73 \\
\hline H. moricandi & 0 & 0 & 0 & 0 & 0 & 0 & 0 & 0 & 0 & 0 & 0 & 16 & 0 & 0 & 0 & 0 & 0 & 0 & 0 & 2 & 113 \\
\hline U. concentricus & 2 & 75 & 2 & 4 & 0 & 8 & 3 & 13 & 32 & 4 & 2 & 46 & 74 & 1 & 3 & 9 & 3 & 1 & 1 & 0 & 610 \\
\hline D. rhuacoicus & 0 & 0 & 0 & 0 & 1 & 0 & 0 & 0 & 1 & 0 & 0 & 0 & 0 & 0 & 0 & 0 & 0 & 0 & 0 & 3 & 13 \\
\hline A. tenebricosus & 0 & 0 & 0 & 0 & 0 & 0 & 0 & 0 & 0 & 0 & 0 & 0 & 0 & 0 & 0 & 0 & 0 & 0 & 0 & 0 & 2 \\
\hline C. fluminea & 0 & 0 & 0 & 0 & 0 & 0 & 0 & 0 & 0 & 0 & 0 & 0 & 0 & 0 & 0 & 0 & 0 & 0 & 0 & 0 & 149 \\
\hline P. punctiferum & 0 & 0 & 0 & 0 & 0 & 0 & 0 & 0 & 0 & 0 & 0 & 0 & 0 & 0 & 0 & 0 & 0 & 0 & 1 & 0 & 1 \\
\hline P. sterkianum & 0 & 0 & 2 & 0 & 0 & 0 & 0 & 0 & 0 & 0 & 0 & 0 & 0 & 0 & 0 & 0 & 0 & 0 & 0 & 0 & 54 \\
\hline E. elliptica & 0 & 0 & 2 & 0 & 0 & 0 & 0 & 0 & 0 & 0 & 0 & 0 & 0 & 0 & 0 & 0 & 0 & 0 & 0 & 0 & 2 \\
\hline E. klappenbachi & 0 & 0 & 0 & 0 & 0 & 0 & 0 & 0 & 0 & 0 & 0 & 0 & 0 & 0 & 0 & 0 & 0 & 0 & 0 & 1 & 18 \\
\hline $\mathbf{S}$ & 1 & 1 & 6 & 1 & 1 & 1 & 2 & 2 & 6 & 1 & 1 & 6 & 1 & 1 & 1 & 2 & 2 & 1 & 4 & 6 & 2300 \\
\hline $\mathbf{N}$ & 2 & 75 & 57 & 4 & 1 & 8 & 26 & 14 & 296 & 4 & 2 & 323 & 74 & 1 & 3 & 10 & 5 & 1 & 12 & 182 & 2300 \\
\hline
\end{tabular}

As espécies dominantes na área de estudo foram os gastrópodes $U$. concentricus $(26 \%)$ e P. catharinae (21\%). Entre os bivalves, a espécie invasora asiática, C. fluminea, foi dominante (62\%). Uncancylus concentricus teve a ocorrência mais ampla, sendo encontrada em $81 \%$ dos locais. Corbicula fluminea, apesar de abundante, teve ocorrência restrita a um único local de coleta, enquanto E. klapppenbachi ocorreu em apenas dois locais. Já P. americanista, P. punctiferum, E. elliptica e A. tenebricosus foram, também, raras, sendo representadas por no máximo três indivíduos.

2000a, Mansur \& Pereira 2006, Pereira et al. 2011). Como será discutido adiante, essa condição está relacionada à variação do relevo e da geologia da região estudada, de montante para jusante.

De maneira geral, as espécies dominantes e melhor distribuídas nos riachos da Bacia do Rio Toropi refletem adequadamente o predomínio de cascalho e a presença localizada e pouco diversificada de macrófitas da região de estudo. Potamolithus catharinae e $U$. concentricus são comuns nos rios de encosta de regiões próximas, com substrato cascalhoso (Veiteinheimer-Mendes et al. 1992, Pereira et al. 2011). Na bacia do Rio Toropi, P. catharinae assim como $P$. ribeirensis predominam em riachos de $4^{\mathrm{a}}$ ordem e nos locais situados em áreas de maior altitude, onde a velocidade da água e o conteúdo de oxigênio costumam ser maiores. Ambas são espécies de prosobrânquios e dependem de águas bem oxigenadas 

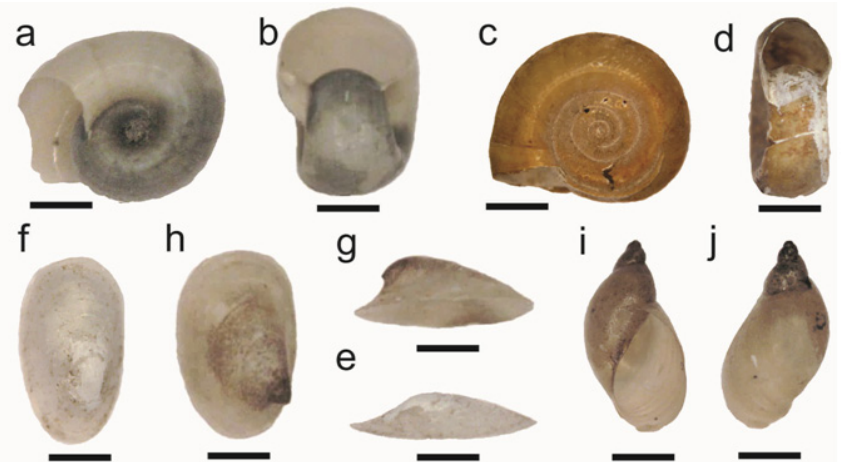

e
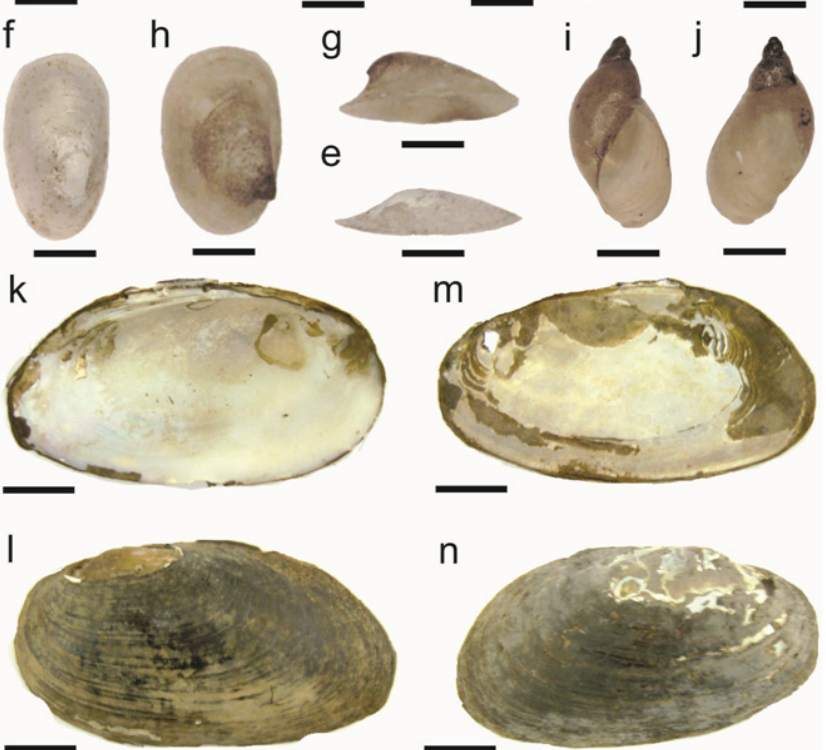

Figura 4. Biomphalaria straminea $(\mathrm{a}, \mathrm{b})$ vista lateral direita e ventral $($ escala $=0,1 \mathrm{~cm}) ;$ Biomphalaria tenagophila $(\mathrm{c}, \mathrm{d})$ vista lateral direita e ventral (escala $=0,3 \mathrm{~cm})$; Hebetancylus moricandi $(\mathrm{e}, \mathrm{f})$ vista lateral e dorsal (escala $=0,5 \mathrm{~cm})$; Uncancylus concentricus $(\mathrm{g}, \mathrm{h})$ vista lateral e dorsal (escala $=0,2 \mathrm{~cm})$; Lymnaea columella $(\mathrm{i}, \mathrm{j})$ vista ventral e dorsal (escala $=0,3 \mathrm{~cm})$; Diplodon rhuacoicus $(\mathrm{k}, 1)$ vista interna e externa $($ escala $=1 \mathrm{~cm}) ;$ Anodontites tenebricosus $(\mathrm{m}, \mathrm{n})$ vista interna e externa $($ escala $=1 \mathrm{~cm})$.

Figure 4. Biomphalaria straminea $(\mathrm{a}, \mathrm{b})$ right side and ventral view $($ scale $=0.1 \mathrm{~cm}) ;$ Biomphalaria tenagophila $(\mathrm{c}, \mathrm{d})$ right side and ventral view (scale $=0.3 \mathrm{~cm})$; Hebetancylus moricandi $(\mathrm{e}, \mathrm{f})$ lateral and dorsal view (scale $=0.5 \mathrm{~cm})$; Uncancylus concentricus $(\mathrm{g}, \mathrm{h})$ lateral and dorsal view $($ scale $=0.2 \mathrm{~cm})$; Lymnaea columella $(\mathrm{i}, \mathrm{j})$ ventral and dorsal view $($ scale $=0.3 \mathrm{~cm})$; Diplodon rhuacoicus $(\mathrm{k}, 1)$ internal and external view $(\mathrm{scale}=1 \mathrm{~cm})$; Anodontites tenebricosus $(\mathrm{m}, \mathrm{n})$ internal and external view $($ scale $=1 \mathrm{~cm})$.

para respirar (Brown et al. 1998). Hebetancylus moricandi foi abundante apenas em um riacho de $4^{\mathrm{a}}$ ordem, situado em área com baixa altitude, com substrato lamoso e mácrofitas. Esta espécie tem sido encontrada, comumente, em ambientes não poluídos, vivendo preferencialmente aderida a folhas ou talos de plantas aquáticas e folhas em decomposição (Pfeifer \& Pitoni 2003, Santos 2003, Martello et al. 2006). Já U. concentricus mostrou tendência a ocorrer em áreas mais altas da encosta, em locais com substrato cascalhoso. Além de ser encontrada em substrato cascalhoso, esta espécie vive preferencialmente associada à macrófitas, madeiras e galhos secos (Lanzer \& Veitenheimer-Mendes 1985, Pereira et al. 2011).

O único bivalve abundante na área de estudo, a espécie invasora C. fluminea, é um filtrador eficiente (Strayer et al. 1999, Spooner \& Vaughn 2006). Riachos de ordem média, localizados em áreas mais baixas, tem maior conteúdo de partículas finas, as quais favorecem a presença de organismos filtradores (Vannote et al. 1980). Por isso, apesar de abundante na área de estudo, C. fluminea teve ocorrência restrita a um único local de $4^{\mathrm{a}}$ ordem e baixa altitude com predomínio de areia. Bivalves Unionoida (D. rhuacoicus e A. tenebricosus), embora raros na área de estudo, também ocorreram em riachos de ordem média $\left(3^{a}\right.$ e $\left.4^{a}\right)$ e em locais com altitude mais baixa. Mexilhões
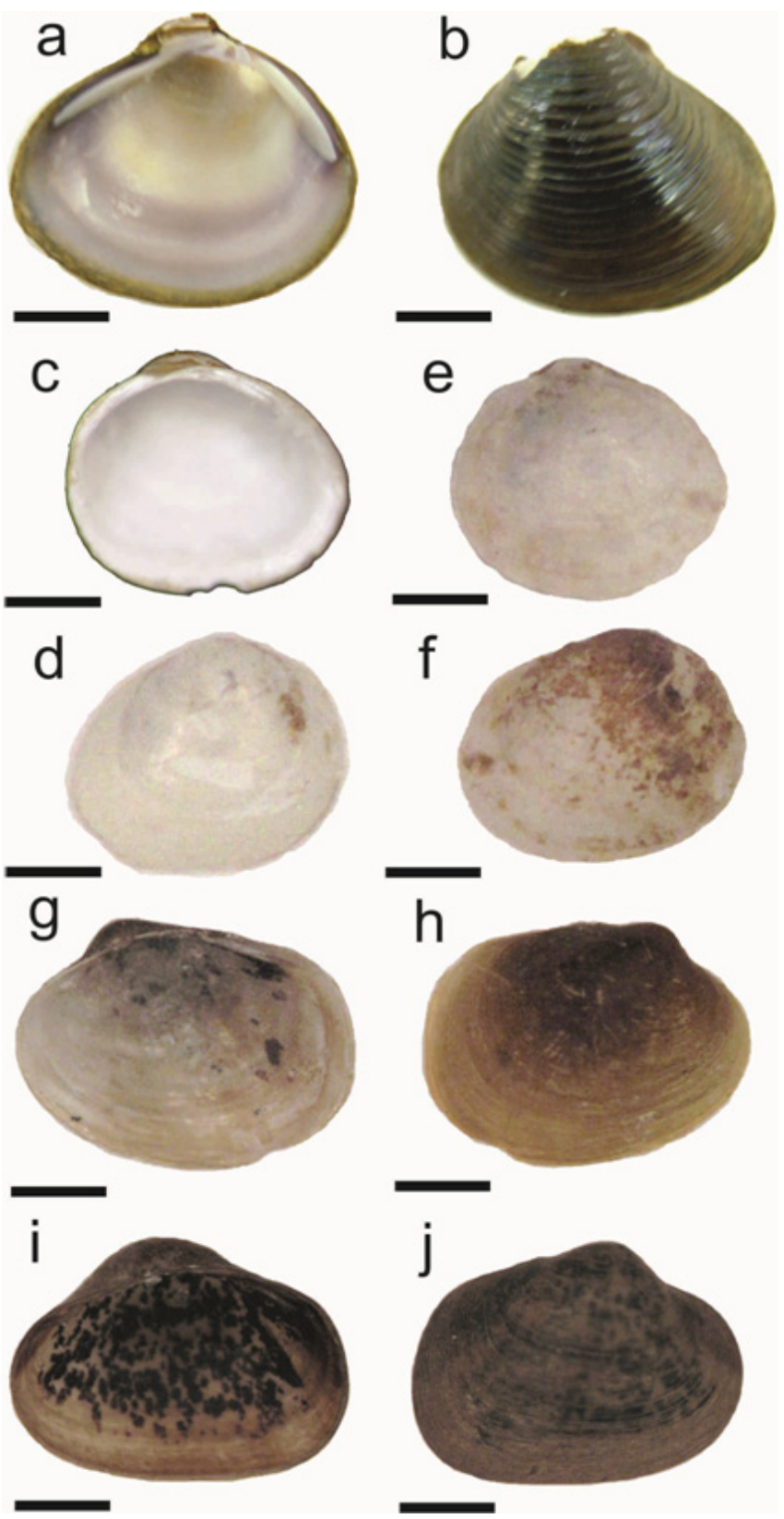

Figura 5. Corbicula fluminea $(\mathrm{a}, \mathrm{b})$ vista interna e externa (escala $=0,7 \mathrm{~cm})$; Pisidium punctiferum $(\mathrm{c}, \mathrm{d})$ vista interna e externa (escala $=0,05 \mathrm{~cm})$; Pisidium sterkianum $(\mathrm{e}, \mathrm{f})$ vista interna e externa (escala $=0,05 \mathrm{~cm})$; Eupera elliptica $(\mathrm{g}, \mathrm{h})$ vista interna e externa (escala $=0,1 \mathrm{~cm})$; Eupera klappenbachi $(\mathrm{i}, \mathrm{j})$ vista interna e externa $($ escala $=0,1 \mathrm{~cm})$.

Figure 5. Corbicula fluminea $(\mathrm{a}, \mathrm{b})$ internal and external view $(\mathrm{scale}=0.7 \mathrm{~cm})$; Pisidium punctiferum $(\mathrm{c}, \mathrm{d})$ internal and external view $(\mathrm{scale}=0.05 \mathrm{~cm})$; Pisidium sterkianum $(\mathrm{e}, \mathrm{f})$ internal and external view $(\mathrm{scale}=0.05 \mathrm{~cm})$; Eupera elliptica $(\mathrm{g}, \mathrm{h})$ internal and external view $(\mathrm{scale}=0.1 \mathrm{~cm})$; Eupera klappenbach $(\mathrm{i}, \mathrm{j})$ internal and external view $($ scale $=0.1 \mathrm{~cm})$

de água doce, apesar de filtradores, só são abundantes e diversificados no sul do Brasil em segmentos de grande ordem de rios como Jacuí (Pfeifer \& Pitoni 2003), dos Sinos (Mansur \& Pereira 2006) e Uruguai (Castillo et al. 2007), refletindo um comportamento geral do grupo (Strayer et al. 1999).

Moluscos raros ou com ocorrência muito localizada na região aqui estudada, como E. klapppenbachi e $P$. punctiferum, são associados a substratos arenosos e maior riqueza de macrófitas (Pereira et al. 
2000b, Pfeifer \& Pitoni 2003). Desta forma, também refletem as características da área de estudo.

Um estudo conduzido em riachos do curso médio da Bacia do Rio Ibicuí, em área de planície na Depressão Central (Freitas 2011) e, realizado com a mesma metodologia utilizada no presente inventário, obteve resultados diferentes ao que foi obtido na área de encosta do curso superior desta bacia (Bacia do Rio Toropi). No curso médio, as espécies dominantes foram o gastrópode $U$. concentricus e o bivalve Pisidium sp. Além disso, os bivalves predominaram em número de exemplares, em relação aos gastrópodes. Esta diferença deve estar relacionada à granulometria do substrato. De maneira geral, a velocidade dos rios tende a diminuir nas áreas mais baixas (Allan 1997), permitindo a deposição de sedimentos mais finos (Bonetto \& Di Persia 1975, Vannote et al. 1980). Nessas áreas, também ocorrem planícies de inundação que comumente apresentam ambientes lênticos, como remansos, banhados, meandros, etc. (McCabe 2010). Os riachos das regiões de baixa altitude da Bacia do Rio Ibicuí seguem essa tendência, apresentando maior porcentagem de areia do que cascalho (Dantas et al. 2010, Freitas 2011). Como já comentado, maior predominância de finos é uma condição mais apropriada para ocorrência de bivalves filtradores ou que se alimentam de detritos (Vannote et al. 1980, Allan 1997, Allen \& Vaughn 2010). Por outro lado, substratos constituídos por cascalho são mais comuns na região da Encosta do Planalto formada, em boa parte, pelos basaltos da Formação Serra Geral (Kämpf \& Streck 2010). Substrato pedregoso é importante para a ocorrência de muitos gastrópodes, devido ao hábito raspador que apresentam (Pereira et al. 2011), e essa condição deve ter determinado a maior riqueza de gastrópodes na área de encosta aqui estudada. Além disso, as áreas mais baixas amostradas localizavam-se em área de transição de relevo, o que também favorece o aumento da riqueza de macroinvertebrados (Morrone 2006, Principe $\&$ Corigliano 2006). Dessa forma, pode-se dizer que na Bacia do Rio Ibicuí a estrutura das comunidades de moluscos de riachos das regiões de baixa altitude difere daquela que ocorre em riachos de regiões de encosta (Bacia do Rio Toropi).

\section{Conclusões}

A baixa riqueza da comunidade de moluscos registrada nos riachos da bacia do Rio Toropi está relacionada à geomorfologia da região de estudo. Por estar em área de encosta e fluir sobre basalto, o leito dos riachos possui substrato predominantemente cascalhoso com escassez de macrófitas e de partículas orgânicas e inorgânicas finas. Dessa forma, bivalves são raros, especialmente os Unionoida, devido ao hábito filtrador e escavador da maioria das espécies. Nem mesmo o bivalve invasor Corbicula fluminea encontra-se bem distribuído. O predomínio de gastrópodes, cujas espécies geralmente têm hábito raspador e são mais bem adaptadas ao substrato cascalhoso da região, contrasta com o que é observado nos riachos de planície de áreas próximas, nos quais sedimentos arenosos e macrófitas aquáticas ocorrem de forma mais expressiva. Nesses, os bivalves predominam. Embora apresente riqueza menor do que a observada em outros rios e riachos do sul do Brasil, a malacofauna dos riachos da bacia do Rio Toropi exibe aspectos da estrutura das comunidades peculiares. As espécies Potamolithus catharinae e Uncancylus concentricus são dominantes, refletindo a boa correnteza e oxigenação das águas. As peculiaridades da estrutura das comunidades de moluscos mostram que a Encosta do Planalto do Rio Grande do Sul é uma área que deve receber atenção, por parte de programas de proteção ambiental voltados à conservação da integridade dos rios brasileiros.

\section{Agradecimentos}

Os autores são gratos à Dra . Maria Cristina Dreher Mansur (Universidade Federal do Rio Grande do Sul, UFRGS) e à Dr ${ }^{\text {a }}$. Rosane
Lanzer (Universidade de Caxias do Sul, UCS) pela identificação dos Pisidiidae e Ancylidae, respectivamente. Agradecimentos especiais à Andréa Salvarrey, Bruna M. Braun, Elisangela Secretti, Mateus M. Pires, Michelle Antunes, Nicolas Figueiredo, Sarah Freitas e Vanessa Baptista pela ajuda durante o trabalho de campo, e aos revisores anônimos pelas sugestões apresentadas.

\section{Referências Bibliográficas}

AGUDO-PADRÓN, A.I. 2009. Recent terrestrial and freshwater molluscs of Rio Grande do Sul State, RS, Southern Brazil Region: a comprehensive synthesis and check list. Visaya. 1-13.

ALLAN, J.D. 1997. Stream ecology: structure and function of running waters. Chapman \& Hall, London, 436p.

ALLAN, J.D. 2004. Landscapes and Riverscapes: The Influence of Land Use on Stream Ecosystems. Annu. Rev. Ecol. Evol. S. 35:257-84. http:// dx.doi.org/10.1146/annurev.ecolsys.35.120202.110122

ALLAN, J.D. \& FLECKER, A.S. 1993. Biodiversity Conservation in Running Waters: Identifying the major factors that threaten destruction of riverine species and ecosystems. BioScience 43(1):32-43. http://dx.doi. org/10.2307/1312104

ALLEN, D.C. \& VAUGHN, C.C. 2010. Complex hydraulic and substrate variables limit freshwater mussel species richness and abundance. J. N. Am. Benthol. Soc. 29(2):383-394. http://dx.doi.org/10.1899/09-024.1

BESKOW, P.R. 1984. A formação da economia arrozeira do Rio Grande do Sul. Ensaios FEE 4(2):55-84.

BOGAN, A.E. 2008. Global diversity of freshwater mussels (Mollusca, Bivalvia) in freshwater. Hydrobiologia 595:139-147. http://dx.doi. org/10.1007/s10750-007-9011-7

BONETTO, A.A. \& DI PERSIA, D.H. 1975. Las poblaciones de pelecípodos del arroyo Ayuí Grande (Prov. Entre Ríos) y los factores que regulan su distribución y estructura. Ecosur 3:123-151.

BROWN, K.M, ALEXANDER, J.E. \& THORP, J.H. 1998. Differences in the ecology and distribution of lotic pulmonate and prosobranch gastropods. Am. Malacol. Bull. 14:91-101.

BURIOL, G.A., ESTEFANEL, V., CHAGAS, A.C. \& EBERHARDT, D. 2007. Clima e vegetação natural do Estado do Rio Grande do Sul segundo o diagrama climático de Walter e Lieth. Cienc. Florest. 2:91-100.

CASTILLO, A.R., BRASIL, L.G., QUEROL, E., QUEROL, M.V.M., OLIVEIRA, E.V. \& MANSUR, M.C.D. 2007. Moluscos bivalves da localidade de São Marcos, bacia do Médio rio Uruguai, Uruguaiana, Brasil. Rev. Biotemas 20(4):73-79.

COLEMAN, B.D. 1981. On random placement and species-area relations. Math. Biosci. 54:191-215. http://dx.doi.org/10.1016/00255564(81)90086-9

COLWELL, R. \& CODDINGTON, J.A. 1994. Estimating terrestrial biodiversity through extrapolation. Philos. T. Roy. Soc. B. 45:101-118. PMid:7972351. http://dx.doi.org/10.1098/rstb.1994.0091

COWELL, F.A., 2006. "Microeconomics: Principles and Analysis," OUP Catalogue, Oxford University Press.

CUMMINS, R.H. 1994. Taphonomic process in modern freshwater molluscan death assemblages: implications for the freshwater fossil record. Palaeogeogr. Palaeocl. 108:55-73. http://dx.doi.org/10.1016/00310182(94)90022-1

DANTAS, M.E., VIEIRO, A.C. \& SILVA, D.R.A. 2010. Origem das paisagens. In Geodiversidade do Estado do Rio Grande do Sul (A.C. Vieiro \& D.R.A. Silva, orgs.). CPRM, Porto Alegre, 250p.

DORAZIO, R.M. 1999. Design-based and model-based inference in surveys of freshwater mollusks. J. N. Am. Benthol. Soc. 18:118-131. http://dx.doi. org/10.2307/1468012

DOWNING, J.A., VAN METER, P. \& WOOLNOUGH, D.A. 2010. Suspects and evidence: a review of the causes of extirpation and decline in freshwater mussels. Anim. Biodivers. Conserv. 33.2:151-185. 
DRÜGG-HAHN, S., LOPES-PITONI, V., CUNHA, F.B. \& CARVALHO, A.P. 2006. Moluscos límnicos. In Biodiversidade. Regiões da Lagoa do Casamento e dos Butiazais de Tapes, planície costeira do Rio Grande do Sul (F.G. Becker, R.A. Ramos \& L.A. Moura, orgs.). Ministério do Meio Ambiente, Brasília, 388p.

DUDGEON, D., ARTHINGTON, A.H., GESSNER, M.O., KAWABATA, Z.I., KNOWLER, D.J., LÉVÊQUE, C., NAIMAN, R.J., PRIEURRICHARD, A.H., SOTO, D., STIASSNY, M.L.J. \& SULLIVAN, C.A. 2006. Freshwater biodiversity: importance, threats, status and conservation challenges. Biol. Rev. 81:163-182. PMid:16336747. http:// dx.doi.org/10.1017/S1464793105006950

FREITAS, S.L. 2011. Influência da ordem e da microbacia, e de alguns fatores ambientais de escala local, na estrutura e na distribuição espacial de comunidades de moluscos. Trabalho de conclusão de curso, Universidade Federal de Santa Maria, Santa Maria.

HOWARD, J.K. \& CUFFEY, K.M. 2006. The functional role of native freshwater mussels in the fluvial benthic environment. Freshwater Biol. 51:460-474. http://dx.doi.org/10.1111/j.1365-2427.2005.01507.x

HUNDERTMARCK, I.S. \& MIORIN, V.M.F. 2001. Classificação das zonas agroecológicas na sub-bacia Rio Ibicui-Mirim: Adequação das culturas ao tipo de solo- RS. Geografia: Ensino \& Pesquisa 11(1):9-15.

KÄMPF, N. \& STRECK, E.V. 2010. Solos. In Geodiversidade do Estado do Rio Grande do Sul (A.C. Vieiro \& D.R.A. Silva, orgs.). CPRM, Porto Alegre, 250p.

LANZER, R.M. 2001. Distribuição, fatores históricos e dispersão de moluscos límnicos em lagoas no sul do Brasil. Biociências 9(2):63-84.

LANZER, R.M. \& VEITENHEIMER-MENDES, I.L. 1985. Aspectos morfológicos e biológicos de uma população de G. concentrica (Orbigny, 1835) (Mollusca: Ancylidae) de um açude do sul do Brasil. Iheringia Ser. Zool. 65:41-56.

LYSNE, S.J., PEREZ, K.E., BROWN, K.M., MINTON, R.L. \& SIDES, J.D. 2008. A review of freshwater gastropod conservation: challenges and opportunities. J. N. Am. Benthol. Soc. 27:463-470. http://dx.doi. org/10.1899/07-061.1

MALUF, J.R.T. 2000. Nova classificação climática do Estado do Rio Grande do Sul. Revista Brasileira de Agrometeorologia 8:141-150.

MANSUR, M.C.D., HEYDRICH, I., PEREIRA, D., RICHINITTI, L.M.Z., TARASCONI, J.C. \& RIOS, E.C. 2003. Moluscos. In Livro vermelho da fauna ameaçada de extinção no Rio Grande do Sul (C.S. Fontana, G.A. Benck \& R.E. Reis, eds.). EdiPUCRS, Porto Alegre, 632p.

MANSUR, M.C.D., SANTOS, C.P., PEREIRA, D., PAZ, I.C.P., ZURITA, M.L.L., RODRIGUEZ, M.T.R., NEHRKE, M.V. \& BERGONCI, P.E.A. 2012. Moluscos límnicos invasores no Brasil: biologia, prevenção e controle. Porto Alegre: Redes Editora. 418 p.

MANSUR, M.C.D., SCHULZ, C., SILVA, M.G.O. \& CAMPOS-VELHO, N.M.R. 1991. Moluscos bivalves límnicos da Estação Ecológica do Taim e áreas adjacentes, Rio Grande do Sul, Brasil. Iheringia Ser. Zool. 71:43-58.

MANSUR, M.C.D. \& PEREIRA, D. 2006. Bivalves límnicos da bacia do rio dos Sinos, Rio Grande do Sul, Brasil (Bivalvia, Unionoida, Veneroida e Mytiloida). Rev. Bras. Zool. 23(4):1123-1147. http://dx.doi.org/10.1590/ S0101-81752006000400021

MARCUZZO, S., PAGEL, S.M. \& CHIAPETTI, M.I.S. 1998. A Reserva da Biosfera da Mata Atlântica no Rio Grande do Sul: situação atual, ações e perspectivas. Consórcio da Mata Atlântica e Conselho Nacional da Reserva da Biosfera da Mata Atlântica. São Paulo: Cetesb. 48 p.

MARTELLO, A.R., KOTZIAN, C.B. \& SIMÕES, M.G. 2006. Quantitative fidelity of recent freshwater mollusk assemblages from the Touro Passo River, Rio Grande do Sul, Brazil. Iheringia Ser. Zool. 96(4):453-465. http://dx.doi.org/10.1590/S0073-47212006000400010

McCABE, D.J. 2010. Rivers and streams: Life in flowing water. Nature Education Knowledge 1(12):4.
MEHLHOP, P. \& VAUGHN, C.C. 1994. Threats to and Sustainability of ecosystems for Freshwater Mollusks. In: Sustainable Ecological Systems: Implementing an Ecological Approach to Land Management (W. Covington \& L.F. Dehand, eds.). General Technical Report Rm-247 for Rocky Mountain Range and Forest Experimental Station. U.S. Forest Service, U.S. Department of Agriculture, Fort Collins, 363p.

MORRONE, J.J. 2006. Biogeographic areas and transition zones of Latin America and the Caribbean islands based on panbiogeographic and cladistic analyses of the entomofauna. Annu. Rev. Entomol. 51:467-494. PMid:16332220. http://dx.doi.org/10.1146/annurev. ento.50.071803.130447

PEDRON, F.A., POELKING, E.L., DALMOLIN, R.S.D. \& KLAMT, A.C.A. E. 2006. A aptidão de uso da terra como base para o planejamento da utilização dos recursos naturais no município de São João do Polêsine - RS. Ciência Rural 36(1):105-112. http://dx.doi.org/10.1590/ S0103-84782006000100016

PEREIRA, D., ARRUdA, J.O., MENEGAT, R., PORTO, M.L., SCHWARZBOLD, A. \& HARTZ, S.M. 2011. Guildas tróficas, composição e distribuição de espécies de moluscos límnicos no gradiente fluvial de um riacho subtropical brasileiro. Biotemas 1(24):21-36.

PEREIRA, D., KONRAD, H.G. \& PALOSKI, N.I. 2000a. Gastrópodos límnicos da bacia do rio Camaquã, RS, Brasil. Acta Biol. Leopoldensia 22(1):55-66.

PEREIRA, D., VEITENHEIMER-MENDES, I.L., MANSUR, M.C.D. \& SILVA, M.C.P. 2000b. Malacofauna límnica do sistema de irrigação da microbacia do Arroio Capivara, Triunfo, RS, Brasil. Biociências 8(1):137-157.

PÉREZ-QUINTERO, J.C. 2011. Distribution patterns of freshwater mollusks along environmental gradients in the southern Guadiana River Basin (SW Iberian Peninsula). Hydrobiologia 678:65-76. http://dx.doi.org/10.1007/ s10750-011-0821-2

PÉREZ-QUINTERO, J.C. 2012. Environmental determinants of freshwater mollusk biodiversity and identification of priority areas for conservation in Mediterranean water courses. Biodivers. Conserv. 21:3001-3016. http:// dx.doi.org/10.1007/s10531-012-0351-x

PFEIFER, N.T.S. \& PITONI, V.L.L. 2003. Análise qualitativa estacional da fauna de moluscos límnicos no Delta do Jacuí, Rio Grande do Sul, Brasil. Biociências 11(2):145-158.

PRINCIPE, R.E. \& CORIGLIANO, M.C. 2006. Bentic, drifting and marginal macroinvertebrate assemblages in a low river: temporal and spatial variations and size structure. Hydrobiologia 553:303-317. http://dx.doi. org/10.1007/s10750-005-0694-3

QUADROS, F.L. \& PILLAR, V.P. 2002. Transições floresta-campo no Rio Grande do Sul. Ciênc. Ambient. 24:109-118.

RICCIARDI, A., NEVES, R.J. \& RASMUSSEN, J.B. 1998. Impending extinctions of North American freshwater mussels (Unionoida) following the zebra mussel (Dreissena polymorpha) invasion. J. Anim. Ecol. 67: 613-619. http://dx.doi.org/10.1046/j.1365-2656.1998.00220.x

RICCIARDI, A. \& RASMUSSEN, J.B. 1999. Extinction rates of North American freshwater fauna. Conserv. Biol. 13(5): 1220-1222. http:// dx.doi.org/10.1046/j.1523-1739.1999.98380.X

ROBAINA, L.E.S., TRENTIN, R., BAZZAN, T., RECKZIEGEL, E.W., VERDUM, R. \& NARDIN, D. 2010. Compartimentação geomorfológica da bacia hidrográfica do Ibicuí, Rio Grande do Sul, Brasil: proposta de classificação. Revista Brasileira de Geomorfologia 11(2):11-23.

SANTOS, S.B. 2003. Estado atual do conhecimento dos ancilídeos na América do Sul. (Mollusca, Gastropoda, Pulmonata, Basommatophora). Rev. Biol. Trop. 50(3):191-224. 
SANTOS, S.B., THIENGO, S.C., FERNANDEZ, M.A., MIYAHIRA, I.C., GONÇALVES, I.C.B., XIMENES, R.F., MANSUR, M.C.D. \& PEREIRA, D. 2012. Espécies de moluscos límnicos invasores no Brasil. In Moluscos límnicos invasores no Brasil: biologia, prevenção e controle (M.C.D. Mansur, C.P. Santos, D. Pereira, I.C.P. Paz, M.L.L. Zurita, M.T.R. Rodriguez, M.V. Nehrke \& P.E.A. Bergonci, orgs.). Redes Editora, Porto Alegre, 412p.

SILVA, J.L.S., CAMPONOGARA, I., GOMES, J.A.A., FRANTZ, L.C., MORAIS, T.Z. \& KONRAD, C.G. 2006. Mapeamento do Uso e Ocupação da Terra no Município de Toropi-RS, com Sistema de Informação Geográfica. In Congresso Brasileiro de Cadastro Técnico Multifinalitário. UFSC.

SIMONE, L.R.L. 2006. Land and Freshwater Molluscs of Brazil. EGB/ Fapesp, São Paulo, 390p.

SPOONER, D.E. \& VAUGHN, C.C. 2006. Context-dependent effects of freshwater mussels on stream benthic communities. Freshwater Biol. 51:1016-1024. http://dx.doi.org/10.1111/j.1365-2427.2006.01547.x

STRAYER, D.L. 1999. Effects of alien species on freshwater mollusks in North America. J. N. Am. Benthol. Soc. 18:74-98. http://dx.doi. org $/ 10.2307 / 1468010$
STRAYER, D.L., CARACO, N.F., COLE, J.J., FINDLAY, S. \& PACE, M.L. 1999. Transformation of freshwater ecosystems by bivalves: a case study of zebra mussels in the Hudson River. BioScience 49:19-27. http://dx.doi. org/10.2307/1313490

STRONG, E.E., GARGOMINY, O., PONDER, W.F. \& BOUCHET, P. 2008. Global diversity of gastropods (Gastropoda; Mollusca) in freshwater. Hydrobiologia 595:149-166. http://dx.doi.org/10.1007/s10750-0079012-6

TIETZE, E. \& DE FRANCESCO, C.G. 2010. Environmental significance of freshwater mollusks in the Southern Pampas, Argentina: to what detail can local environments be inferred from mollusk composition? Hydrobiologia 641:133-143. http://dx.doi.org/10.1007/s10750-009-0072-7

VANNOTE, R.L., MINSHALL., G.W., CUMMINS, K.W., SEDELL J.R. \& CUSHING, C.E. 1980. The River Continuum Concept. Can. J. Fish. Aquat. Sci. 37:130-137. http://dx.doi.org/10.1139/f80-017

VEITENHEIMER-MENDES, I.L., LOPES-PITONI, V.L., SILVA, M.C.P., ALMEIDA-CAON, J.E. \& SCHRÖDER-PFEIFER, N.T. 1992. Moluscos (Gastropoda e Bivalvia) ocorrentes nas nascentes do rio Gravataí, Rio Grande do Sul, Brasil. Iheringia Ser. Zool. 73:69-76.

Recebido em 05/02/2013 Versão reformulada recebida em 18/04/2013 Publicado em 01/07/2013 